\title{
Incorporation of fermions into double field theory
}

\section{Imtak Jeon, ${ }^{a, 1}$ Kanghoon Lee ${ }^{b}$ and Jeong-Hyuck Park ${ }^{c}$}

${ }^{a}$ CERN, Theory Division, CH-1211 Geneva 23, Switzerland

${ }^{b}$ Center for Quantum Spacetime, Sogang University, Seoul 121-742, Korea

${ }^{c}$ Department of Physics, Sogang University, Seoul 121-742, Korea

E-mail: imtak@sogang.ac.kr, kanghoon@sogang.ac.kr, park@sogang.ac.kr

ABSTRACT: Based on the stringy differential geometry we proposed earlier, we incorporate fermions such as gravitino and dilatino into double field theory in a manifestly covariant manner with regard to $\mathbf{O}(D, D)$ T-duality, diffeomorphism, one-form gauge symmetry for $B$-field and a pair of local Lorentz symmetries. We note that there are two kinds of fermions in double field theory: $\mathbf{O}(D, D)$ singlet and non-singlet which may be identified, respectively as the common and the non-common fermionic sectors in type IIA and IIB supergravities. For each kind, we construct corresponding covariant Dirac operators. Further, we derive a simple criterion for an $\mathbf{O}(D, D)$ rotation to flip the chirality of the $\mathbf{O}(D, D)$ non-singlet chiral fermions, which implies the exchange of type IIA and IIB supergravities.

KeYwords: String Duality, Space-Time Symmetries

ArXiv EPRINT: 1109.2035

\footnotetext{
${ }^{1}$ On leave of absence from Sogang University.
} 


\section{Contents}

1 Introduction $\quad 1$

2 Conventions 4

3 Two types of double-vielbeins and their $\mathrm{O}(D, D)$ transformations $\quad 5$

3.1 Primed and unprimed double-vielbeins 5

$\begin{array}{ll}3.2 \mathbf{O}(D, D) \text { rotations of the double-vielbeins } & 6\end{array}$

4 Covariant Dirac operators $\quad 8$

4.1 Semi-covariant derivative for double-gauge symmetry: review 8

4.2 Unprimed Dirac operators: $\mathbf{O}(D, D)$ singlet 10

$\begin{array}{lll}4.3 & \text { Primed Dirac operators } & 12\end{array}$

4.4 Chirality change under $\mathbf{O}(D, D)$ T-duality 14

$\begin{array}{lll}4.5 & \text { Reduction to } D \text { dimension } & 14\end{array}$

5 Summary and comments $\quad 15$

\section{Introduction}

String theory possesses T-duality and imposes $\mathbf{O}(D, D)$ structure on its $D$-dimensional low energy effective actions [1-4]. The $\mathbf{O}(D, D)$ T-duality can be conveniently described if we formally double the spacetime dimension, from $D$ to $2 D$, with coordinates, $x^{\mu} \rightarrow$ $y^{A}=\left(\tilde{x}_{\mu}, x^{\nu}\right)$. The new coordinates, $\tilde{x}_{\mu}$, may be viewed as the canonical conjugates of the winding modes of closed strings, as noted by Tseytlin and Siegel in the early 90's [5-8]. Recent developments initiated by Hull and Zwiebach developed this idea further, in the name of Double Field Theory (DFT), by writing the $D$-dimensional effective action entirely in terms of the 2D-dimensional language, i.e. $2 D$ tensors [9-12] (see also [13-24]). Yet, as a field theory counterpart to the level matching condition in closed string theories, it is required that all the fields as well as all of their possible products should be annihilated by the $\mathbf{O}(D, D)$ d'Alembert operator, $\partial^{2}=\partial_{A} \partial^{A}$,

$$
\partial^{2} \Phi \equiv 0, \quad \partial_{A} \Phi_{1} \partial^{A} \Phi_{2} \equiv 0 .
$$

This 'level matching constraint' actually means that the theory is not truly doubled: there is a choice of coordinates $\left(\tilde{x}^{\prime}, x^{\prime}\right)$, related to the original coordinates $(\tilde{x}, x)$, by an $\mathbf{O}(D, D)$ rotation, in which all the fields do not depend on the $\tilde{x}^{\prime}$ coordinates [11]. Henceforth, the equivalence symbol, '”', means an equality up to the constraint (1.1), or simply up to the winding coordinate independency, i.e. $\frac{\partial}{\partial \widetilde{x}_{\mu}} \equiv 0$. 
With the spacetime dimension formally doubled in double field theory, T-duality is realized by an $\mathbf{O}(D, D)$ rotation which acts on the $2 D$-dimensional vector indices of an $\mathbf{O}(D, D)$ covariant tensor in a standard manner,

$$
T_{A_{1} A_{2} \cdots A_{n}} \longrightarrow M_{A_{1}}^{B_{1}} M_{A_{2}}{ }^{B_{2}} \cdots M_{A_{n}}{ }^{B_{n}} T_{B_{1} B_{2} \cdots B_{n}}, \quad M \in \mathbf{O}(D, D),
$$

where the $\mathbf{O}(D, D)$ group is defined by the invariance of a constant metric,

$$
M_{A}{ }^{C} M_{B}{ }^{D} \mathcal{J}_{C D}=\mathcal{J}_{A B}, \quad \mathcal{J}_{A B}:=\left(\begin{array}{ll}
0 & 1 \\
1 & 0
\end{array}\right) .
$$

Without imposing the level matching constraint, the $\mathbf{O}(D, D)$ transformation would naturally correspond to a Noether symmetry of the $2 D$-dimensional field theory. However, with the constraint, the double field theory is, by nature, $D$-dimensional living on a $D$ dimensional hyperplane. As the $\mathbf{O}(D, D)$ transformation then rotates the entire hyperplane, the $\mathbf{O}(D, D)$ rotation acts a priori as a 'duality' rather than a 'Noether symmetry' of the $D$-dimensional theory. After further dimensional reductions, it becomes a Noether symmetry of the reduced action, as can be seen in e.g. [1-3, 25-28].

Further, in DFT the $D$-dimensional diffeomorphism, $x^{\mu} \rightarrow x^{\mu}+\delta x^{\mu}$, and the one-form gauge symmetry of the two-form gauge field, $B_{\mu \nu} \rightarrow B_{\mu \nu}+\partial_{\mu} \Lambda_{\nu}-\partial_{\nu} \Lambda_{\mu}$, are naturally combined into what we may call 'double-gauge symmetry' (denoted by ' $\delta_{X}$ '). By definition, the double-gauge transformation of a double-gauge covariant tensor is generated by the Dorfman derivative or generalized Lie derivative, i.e. " $\delta_{X}=\hat{\mathcal{L}}_{X}$ ", whose definition reads [8, $12,29,32,33]$,

$\hat{\mathcal{L}}_{X} T_{A_{1} \cdots A_{n}}:=X^{B} \partial_{B} T_{A_{1} \cdots A_{n}}+\omega_{T} \partial_{B} X^{B} T_{A_{1} \cdots A_{n}}+\sum_{i=1}^{n}\left(\partial_{A_{i}} X_{B}-\partial_{B} X_{A_{i}}\right) T_{A_{1} \cdots A_{i-1}}{ }^{B}{ }_{A_{i+1} \cdots A_{n}}$.

Here $\omega_{T}$ is the given weight of an $\mathbf{O}(D, D)$ covariant tensor, $T_{A_{1} \cdots A_{n}}$, and $X^{A}$ is the doublegauge symmetry parameter whose half components are for the one-form gauge symmetry and the other half are for the diffeomorphism,

$$
X^{A}=\left(\Lambda_{\mu}, \delta x^{\nu}\right) .
$$

As the generalized Lie derivative differs from the ordinary Lie derivative, the underlying differential geometry of DFT should be beyond Riemann [14, 18, 29-34]. Generally speaking, while the fundamental object in Riemannian geometry is a metric, closed string theories call for us to put the $B$-field and a scalar dilaton on an equal footing with the metric, and hence call for new geometry.

In our previous works $[14,18]$, we proposed a novel differential geometry for double field theory that treats the three objects in a unified manner and manifests $\mathbf{O}(D, D)$ T-duality, the double-gauge symmetry, and also a pair of local Lorentz symmetries simultaneously. The key concept therein is 'semi-covariant derivative' that we review later.

In this paper, utilizing the semi-covariant derivative approach, we incorporate fermions, such as gravitino and dilatino, into double field theory. Especially we construct covariant 
- $\mathbf{O}(D, D)$ T-duality

- Gauge symmetries

1. Double-gauge symmetry

- Diffeomorphism

- One-form gauge symmetry

2. A pair of Local Lorentz symmetries, $\mathbf{S O}(1, D-1) \times \mathbf{S O}(D-1,1)$

Table 1. T-duality and gauge symmetries in DFT.

DFT Dirac operators that are manifestly compatible with all the symmetries in table 1. Upon the level matching constraint (1.1) and in terms of the undoubled $D$-dimensional component fields, our Dirac operators reduce to those found recently by Coimbra, StricklandConstable and Waldram as for the unifying reformulation of type IIA and IIB supergravities [34].

Further we show that there are two kinds of fermions in double field theory:

1. $\mathbf{O}(D, D)$ singlet fermions that, in our notation, consist of 'unprimed' gravitino and dilatino. Their local Lorentz indices (spinorial and vectorial) are singlet under $\mathbf{O}(D, D)$ T-duality. They couple to 'unprimed double-vielbein' [18],

$$
\left(\psi_{\bar{p}}^{\alpha}, \rho^{\alpha}\right) \quad \Longleftrightarrow \quad\left(V_{A p}, \bar{V}_{B \bar{p}}\right) .
$$

The common fermionic sector of type IIA and IIB supergravities may be identified as our unprimed fermions.

2. $\mathbf{O}(D, D)$ non-singlet fermions that consist of 'primed' gravitino and dilatino. Their local Lorentz indices transform nontrivially under $\mathbf{O}(D, D)$ T-duality. They couple to 'primed double-vielbein',

$$
\left(\psi_{\bar{p}}^{\prime \alpha}, \rho^{\alpha}\right) \quad \Longleftrightarrow \quad\left(V_{A \bar{p}}^{\prime}, \bar{V}_{B p}^{\prime}\right)
$$

The non-common fermions of the opposite chiralities in type IIA and IIB supergravities correspond to our primed fermions.

We also present a criterion for $\mathbf{O}(D, D)$ rotations to flip the chirality of the primed fermions, which turns out to depend on both the $\mathbf{O}(D, D)$ group element and the background fields. This generalizes, in a unifying manner, the earlier works by Hassan in 90 's [35-37].

The organization of the present paper is as follows. To start, in section 2 we set up our conventions including the indices used for each representation of the symmetries in table 1 . In section 3, after reviewing the two types of the double-vielbeins from [18], we analyze their finite $\mathbf{O}(D, D)$ transformations. In section 4, utilizing the semi-covariant derivative, we construct the covariant Dirac operators for each type of the fermions and derive the criterion for the primed fermions to flip their chiralities under $\mathbf{O}(D, D)$ T-duality. Section 5 contains the summary and comments. 


\begin{tabular}{|c|c|c|}
\hline indices & representation & metric \\
\hline$A, B, \cdots$ & double-gauge vector & $\mathcal{J}_{A B}$ in eq. (1.3) \\
$p, q, \cdots$ & $\mathbf{S O}(1, D-1)$ vector & $\eta_{p q}=\operatorname{diag}(-++\cdots+)$ \\
$\alpha, \beta, \cdots$ & $\mathbf{S p i n}(1, D-1)$ spinor & $C_{\alpha \beta}$ in eq. $(2.2)$ \\
$\bar{p}, \bar{q}, \cdots$ & $\mathbf{S O}(D-1,1)$ vector & $\bar{\eta}_{\bar{p} \bar{q}}=\operatorname{diag}(+--\cdots-)$ \\
$\bar{\alpha}, \bar{\beta}, \cdots$ & $\mathbf{S p i n}(D-1,1)$ spinor & $\bar{C}_{\bar{\alpha} \bar{\beta}}$ in eq. $(2.2)$ \\
\hline
\end{tabular}

Table 2. Indices used for each symmetry representation and the relevant metrics that raise or lower the positions of them. While $\mathbf{O}(D, D)$ acts always on the double-gauge vector indices (capital Roman), it may also rotate other indices of the primed fields (1.7). It is the characteristic feature of DFT that, although the $\mathbf{O}(D, D)$ metric $\mathcal{J}_{A B}$ (1.3) is a constant 'flat' one, the corresponding 'flat' indices, $A, B, \cdots$, decompose into $D$-dimensional curved spacetime vector and one-form indices, as in (1.5), (3.6), (3.10), etc. [9-12].

\section{Conventions}

In table 2, we summarize our conventions for indices and metrics used for each representation of the symmetries listed in table $1 .{ }^{1}$ For the application of our formalism to type IIA and IIB supergravities, in this paper we focus on 'even' $D$-dimensional Minkowskian spacetime that admits Majorana-Weyl spinors, i.e. $D \approx 2 \bmod 8$.

For the two Minkowskian metrics, $\eta_{p q}$ and $\bar{\eta}_{\bar{p} \bar{q}}$, we introduce separately the corresponding 'real' gamma matrices: $\left(\gamma^{p}\right)^{\alpha}{ }_{\beta}$ and $\left(\bar{\gamma}^{\bar{p}}\right)^{\bar{\alpha}}{ }_{\bar{\beta}}$ satisfying

$$
\begin{array}{ll}
\gamma^{p}=\left(\gamma^{p}\right)^{*}, & \gamma^{p} \gamma^{q}+\gamma^{q} \gamma^{p}=2 \eta^{p q} \\
\bar{\gamma}^{\bar{p}}=\left(\bar{\gamma}^{\bar{p}}\right)^{*}, & \bar{\gamma}^{\bar{p}} \bar{\gamma}^{\bar{q}}+\bar{\gamma}^{\bar{q}} \bar{\gamma}^{\bar{p}}=2 \bar{\eta}^{\bar{p} \bar{q}} .
\end{array}
$$

Their charge conjugation matrices, $C_{\alpha \beta}$ and $\bar{C}_{\bar{\alpha} \bar{\beta}}$, meet ${ }^{2}$

$$
\begin{aligned}
& \left(C \gamma^{p_{1} p_{2} \cdots p_{n}}\right)_{\alpha \beta}=-(-1)^{n(n+1) / 2}\left(C \gamma^{p_{1} p_{2} \cdots p_{n}}\right)_{\beta \alpha}, \\
& \left(\bar{C} \bar{\gamma}^{\bar{p}_{1} \bar{p}_{2} \cdots \bar{p}_{n}}\right)_{\bar{\alpha} \bar{\beta}}=-(-1)^{n(n+1) / 2}\left(\bar{C} \bar{\gamma}^{\bar{p}_{1} \bar{p}_{2} \cdots \bar{p}_{n}}\right)_{\bar{\beta} \bar{\alpha}},
\end{aligned}
$$

and define the charge-conjugated spinors. For the unprimed and primed $\operatorname{Spin}(1, D-1)$ spinors we have

$$
\bar{\psi}_{\bar{p} \alpha}=\psi_{\bar{p}}^{\beta} C_{\beta \alpha}, \quad \bar{\rho}_{\alpha}=\rho^{\beta} C_{\beta \alpha}, \quad \bar{\psi}_{\bar{p} \alpha}^{\prime}=\psi_{\bar{p}}^{\prime \beta} C_{\beta \alpha}, \quad \bar{\rho}_{\alpha}^{\prime}=\rho^{\prime \beta} C_{\beta \alpha} .
$$

We also set, in order to specify the chirality of the Weyl spinors,

$$
\gamma^{(D+1)}:=\gamma^{012 \cdots D-1}, \quad \bar{\gamma}^{(D+1)}:=\bar{\gamma}^{012 \cdots D-1},
$$

that satisfy

$$
\gamma^{p} \gamma^{(D+1)}+\gamma^{(D+1)} \gamma^{p}=0, \quad\left(\gamma^{(D+1)}\right)^{2}=1, \quad \bar{\gamma}^{\bar{p}} \bar{\gamma}^{(D+1)}+\bar{\gamma}^{(D+1)} \bar{\gamma}^{\bar{p}}=0, \quad\left(\bar{\gamma}^{(D+1)}\right)^{2}=1 .
$$

\footnotetext{
${ }^{1}$ Note the opposite signatures chosen for $\eta$ and $\bar{\eta}$, i.e. mostly plus vs. mostly minus (cf. [18]).

${ }^{2} \mathrm{~A}$ possible relation between the unbarred and barred real gamma matrices is to identify $\bar{\gamma}^{\bar{p}}$ with $\gamma^{p} \gamma^{(D+1)}$, and $\bar{C}$ with $C$. However, we do not need to impose this identification in the present paper.
} 
The unprimed fermions, $\left(\psi_{\bar{p}}^{\alpha}, \rho^{\alpha}\right)$, are set to be Majorana-Weyl spinors of the fixed chiralities,

$$
\gamma^{(D+1)} \psi_{\bar{p}}=+\psi_{\bar{p}}, \quad \gamma^{(D+1)} \rho=-\rho .
$$

On the other hand, the primed fermions, $\left(\psi^{\prime} \overline{\bar{p}}, \rho^{\prime \alpha}\right)$, are Majorana-Weyl spinors possessing either the same chirality (as for type IIB supergravity),

$$
\gamma^{(D+1)} \psi_{\bar{p}}^{\prime}=+\psi_{\bar{p}}^{\prime}, \quad \gamma^{(D+1)} \rho^{\prime}=-\rho^{\prime},
$$

or the opposite chirality (as for type IIA supergravity),

$$
\gamma^{(D+1)} \psi_{\bar{p}}^{\prime}=-\psi_{\bar{p}}^{\prime}, \quad \gamma^{(D+1)} \rho^{\prime}=+\rho^{\prime} .
$$

The chiralities of the primed fermions may be flipped under $\mathbf{O}(D, D)$ T-duality, as we shall see later.

\section{Two types of double-vielbeins and their $\mathrm{O}(D, D)$ transformations}

\subsection{Primed and unprimed double-vielbeins}

There are two types of vielbeins in DFT [18]. We distinguish them here as unprimed doublevielbein, $\left(V_{A p}, \bar{V}_{B \bar{q}}\right)$, and primed double-vielbein, ${ }^{3}\left(V_{A \bar{p}}^{\prime} \bar{V}_{B q}^{\prime}\right)$. They carry opposite local Lorentz vector indices.

In terms of the flat metrics in table 2, the unprimed double-vielbein satisfies the following defining properties [18]:

$$
V_{A p} V_{q}^{A}=\eta_{p q}, \quad V_{A p} \bar{V}_{\bar{q}}^{A}=0, \quad \bar{V}_{A \bar{p}} \bar{V}_{\bar{q}}^{A}=\bar{\eta}_{\bar{p} \bar{q}}, \quad V_{A p} V_{B}^{p}+\bar{V}_{A \bar{p}} \bar{V}_{B}^{\bar{p}}=\mathcal{J}_{A B} .
$$

Hence the double-vielbein forms a pair of rank-two projections [14],

$$
P_{A B}:=V_{A}^{p} V_{B p}, \quad \bar{P}_{A B}:=\bar{V}_{A} \bar{p}^{\bar{p}} \bar{V}_{B \bar{p}},
$$

that are symmetric, orthogonal and complementary to each other,

$$
\begin{aligned}
P_{A B} & =P_{B A}, & \bar{P}_{A B} & =\bar{P}_{B A}, & P_{A}{ }^{B} \bar{P}_{B}{ }^{C} & =0, \\
P_{A}{ }^{B} P_{B}{ }^{C} & =P_{A}^{C}, & \bar{P}_{A}{ }^{B} \bar{P}_{B}^{C} & =\bar{P}_{A}^{C}, & P_{A}^{B}+\bar{P}_{A}^{B} & =\delta_{A}^{B},
\end{aligned}
$$

and further meet

$$
P_{A}^{B} V_{B p}=V_{A p}, \quad \bar{P}_{A}^{B} \bar{V}_{B \bar{p}}=\bar{V}_{A \bar{p}}, \quad \bar{P}_{A}^{B} V_{B p}=0, \quad P_{A}^{B} \bar{V}_{B \bar{p}}=0 .
$$

The defining properties of the double-vielbein (3.1) actually means then that, as a $2 D \times 2 D$ matrix, $\left(V_{A}^{p}, \bar{V}_{B} \bar{q}\right)$ diagonalize both the projectors $P_{A B}$ and $\bar{P}_{A B}$, or equivalently both the $\mathbf{O}(D, D)$ metric $\mathcal{J}_{A B}$ and the "generalized metric" $\mathcal{H}_{A B}:=(P-\bar{P})_{A B}$, as follows [18],

$$
\mathcal{J}=(V, \bar{V})\left(\begin{array}{ll}
\eta & 0 \\
0 & \bar{\eta}
\end{array}\right)(V, \bar{V})^{t}, \quad \mathcal{H}=(V, \bar{V})\left(\begin{array}{cc}
\eta & 0 \\
0 & -\bar{\eta}
\end{array}\right)(V, \bar{V})^{t} .
$$

\footnotetext{
${ }^{3}$ In [18], the latter was called "twin double-vielbein".
} 
Assuming that the upper half blocks are non-degenerate, the unprimed double-vielbein takes the following most general form $[18],{ }^{4}$

$$
V_{A p}=\frac{1}{\sqrt{2}}\left(\begin{array}{c}
\left(e^{-1}\right)_{p}{ }^{\mu} \\
(B+e)_{\nu p}
\end{array}\right), \quad \bar{V}_{A \bar{p}}=\frac{1}{\sqrt{2}}\left(\begin{array}{c}
\left(\bar{e}^{-1}\right)_{\bar{p}}{ }^{\mu} \\
(B+\bar{e})_{\nu \bar{p}}
\end{array}\right) .
$$

Here $e_{\mu}{ }^{p}$ and $\bar{e}_{\nu}{ }^{\bar{p}}$ are two copies of the $D$-dimensional vielbein corresponding to the same spacetime metric in the following manner,

$$
e_{\mu}^{p} e_{\nu}^{q} \eta_{p q}=-\bar{e}_{\mu}{ }^{\bar{p}} \bar{e}_{\nu} \bar{q}_{\bar{\eta}} \bar{\eta}_{\bar{q} q}=g_{\mu \nu}
$$

and $B_{\mu \nu}$ corresponds to the Kalb-Ramond two-form gauge field. We also set in (3.6),

$$
B_{\mu p}=B_{\mu \nu}\left(e^{-1}\right)_{p}{ }^{\nu}, \quad B_{\mu \bar{p}}=B_{\mu \nu}\left(\bar{e}^{-1}\right)_{\bar{p}}{ }^{\nu}
$$

In particular, $\left(\bar{e}^{-1} e\right)_{\bar{p}}^{p}$ and $\left(e^{-1} \bar{e}\right)_{p} \bar{p}$ are local Lorentz transformations,

$$
\left(\bar{e}^{-1} e\right)_{\bar{p}}^{p}\left(\bar{e}^{-1} e\right)_{\bar{q}}^{q} \eta_{p q}=-\bar{\eta}_{\bar{p} \bar{q}}, \quad\left(e^{-1} \bar{e}\right)_{p} \bar{p}\left(e^{-1} \bar{e}\right)_{q}{ }_{q} \bar{\eta}_{\bar{p} \bar{q}}=-\eta_{p q}
$$

Now, having the explicit form of the unprimed double-vielbein (3.6), we are able to define the 'primed double-vielbein',

$$
V_{A \bar{p}}^{\prime}:=\left(\bar{e}^{-1} e\right)_{\bar{p}}{ }^{p} V_{A p}=\frac{1}{\sqrt{2}}\left(\begin{array}{c}
\left(\bar{e}^{-1}\right)_{\bar{p}}^{\mu} \\
(B-\bar{e})_{\nu \bar{p}}
\end{array}\right), \quad \bar{V}_{A p}^{\prime}:=\left(e^{-1} \bar{e}\right)_{p} \bar{p} \bar{V}_{A \bar{p}}=\frac{1}{\sqrt{2}}\left(\begin{array}{c}
\left(e^{-1}\right)_{p}{ }^{\mu} \\
(B-e)_{\nu p}
\end{array}\right) .
$$

They satisfy, parallel to (3.1),

$$
V_{A \bar{p}}^{\prime} V_{\bar{q}}^{\prime A}=-\bar{\eta}_{\bar{p} \bar{q}}, \quad V_{A \bar{p}}^{\prime} \bar{V}_{q}^{\prime A}=0, \quad \bar{V}_{A p}^{\prime} \bar{V}^{\prime A}{ }_{q}=-\eta_{p q}, \quad V_{A \bar{p}}^{\prime} V_{B}^{\prime}{ }_{B}^{\bar{p}}+\bar{V}_{A p}^{\prime} \bar{V}_{B}^{\prime}{ }^{p}=-\mathcal{J}_{A B},
$$

and

$$
\begin{aligned}
-V_{A}^{\prime}{ }_{A}^{\bar{p}} V_{B \bar{p}}^{\prime} & =V_{A}{ }^{p} V_{B p}=P_{A B}, & -\bar{V}_{A}^{\prime}{ }_{A} \bar{V}_{B p}^{\prime} & =\bar{V}_{A}{ }^{\bar{p}} \bar{V}_{B \bar{p}}=\bar{P}_{A B}, \\
P_{A}{ }^{B} V_{B \bar{p}}^{\prime} & =V_{A \bar{p}}^{\prime}, & \bar{P}_{A}{ }^{B} \bar{V}_{B p}^{\prime} & =\bar{V}_{A p}^{\prime} .
\end{aligned}
$$

\section{$3.2 \mathrm{O}(D, D)$ rotations of the double-vielbeins}

Both the primed and unprimed double-vielbeins are covariant with respect to the local Lorentz symmetries and the double-gauge symmetry i.e. " $\delta_{X} \equiv \hat{\mathcal{L}}_{X}$ ". What make them distinguishable are their $\mathbf{O}(D, D)$ T-duality transformations. Once we set the unprimed double-vielbein to be a covariant $\mathbf{O}(D, D)$ vector,

$$
V_{A p} \longrightarrow M_{A}^{B} V_{B p}, \quad \bar{V}_{A \bar{p}} \longrightarrow M_{A}^{B} \bar{V}_{B \bar{p}}
$$

\footnotetext{
${ }^{4}$ It is worth while to note that (up to the upper half block non-degeneracy assumption), (3.6) is the most general form of the double-vielbein parametrization that diagonalizes both the $\mathbf{O}(D, D)$ metric, $\mathcal{J}_{A B}$, and the generalized metric, $\mathcal{H}_{A B}$, as in (3.5). For other parametrization that diagonalizes $\mathcal{H}_{A B}$ only, see the early work by Maharana and Schwarz [27].
} 
the primed double-vielbein cannot transform as an $\mathbf{O}(D, D)$ vector: Its Lorentz vector indices must be rotated too, as first noted in [18] for infinitesimal $\mathbf{O}(D, D)$ transformations. Below we analyze their 'finite' $\mathbf{O}(D, D)$ transformations, for later discussion on the chirality change of the primed fermions under $\mathbf{O}(D, D)$ T-duality.

If we explicitly parametrize a generic $\mathbf{O}(D, D)$ element as

$$
M_{A}{ }^{B}=\left(\begin{array}{ll}
\mathbf{a}^{\mu}{ }_{\nu} & \mathbf{b}^{\mu \sigma} \\
\mathbf{c}_{\rho \nu} & \mathbf{d}_{\rho}{ }^{\sigma}
\end{array}\right),
$$

the defining property of the $\mathbf{O}(D, D)$ group (1.3) implies

$$
\mathbf{a b} \mathbf{b}^{t}+\mathbf{b a}^{t}=0, \quad \mathbf{c d}^{t}+\mathbf{d c}^{t}=0, \quad \mathbf{a d}^{t}+\mathbf{b c}^{t}=1 .
$$

From the vectorial $\mathbf{O}(D, D)$ transformation rule (3.13) of the unprimed double-vielbein (3.6), we note, among others,

$$
e^{-1} \longrightarrow e^{-1}\left[\mathbf{a}^{t}+(g-B) \mathbf{b}^{t}\right], \quad \bar{e}^{-1} \longrightarrow \bar{e}^{-1}\left[\mathbf{a}^{t}-(g+B) \mathbf{b}^{t}\right],
$$

and hence

$$
\left(e^{-1} \bar{e}\right)_{p}^{\bar{p}} \longrightarrow L_{p}^{q}\left(e^{-1} \bar{e}\right)_{q}^{\bar{p}}, \quad\left(\bar{e}^{-1} e\right)_{\bar{p}}^{p} \quad \longrightarrow \quad \bar{L}_{\bar{p}}^{\bar{q}}\left(\bar{e}^{-1} e\right)_{\bar{q}}^{p}
$$

where we set

$$
L=e^{-1}\left[\mathbf{a}^{t}+(g-B) \mathbf{b}^{t}\right]\left[\mathbf{a}^{t}-(g+B) \mathbf{b}^{t}\right]^{-1} e, \quad \bar{L}=\left(\bar{e}^{-1} e\right) L^{-1}\left(e^{-1} \bar{e}\right) .
$$

The crucial properties of $L$ and $\bar{L}$ are that they are local Lorentz transformations,

$$
L_{p}^{r} L_{q}^{s} \eta_{r s}=\eta_{p q}, \quad \bar{L}_{\bar{p}} \bar{r}^{\bar{r}} \bar{L}_{\bar{q}}{ }^{s} \bar{\eta}_{\bar{r} \bar{s}}=\bar{\eta}_{\bar{p} \bar{q}}
$$

These can be verified directly from (3.15) and

$$
[\mathbf{a}+\mathbf{b}(g+B)] g^{-1}\left[\mathbf{a}^{t}+(g-B) \mathbf{b}^{t}\right]=[\mathbf{a}-\mathbf{b}(g-B)] g^{-1}\left[\mathbf{a}^{t}-(g+B) \mathbf{b}^{t}\right] .
$$

In fact, from the consideration that $\left(e^{-1} \bar{e}\right)_{p} \bar{p}$ and $\left(\bar{e}^{-1} e\right)_{\bar{p}}^{p}$ themselves are local Lorentz transformations and also that this property must be preserved under $\mathbf{O}(D, D)$ T-duality, it follows naturally that $L$ and $\bar{L}$ must correspond to local Lorentz transformations.

Therefore, under $\mathbf{O}(D, D)$ T-duality the primed double-vielbein transforms nontrivially as

$$
V_{A \bar{p}}^{\prime} \longrightarrow M_{A}^{B} \bar{L}_{\bar{p}}^{\bar{q}} V_{B \bar{q}}^{\prime}, \quad \bar{V}_{A p}^{\prime} \longrightarrow M_{A}^{B} L_{p}^{q} \bar{V}_{B q}^{\prime}
$$

where $L$ and $\bar{L}$ are local Lorentz transformations (3.18) depending on both the $\mathbf{O}(D, D)$ element, $M$, and the backgrounds, $g_{\mu \nu}, B_{\mu \nu}$. 
It is well known that even-dimensional irreducible gamma matrices are unique up to similarity transformations, essentially due to Schur's lemma. This implies, for the cases of (2.1), (3.9) and (3.19), that there must be similarity transformations, $S_{e}$ and $S_{L}$ relating

$$
\gamma^{p}\left(e^{-1} \bar{e}\right)_{p}^{\bar{p}}=S_{e} \bar{\gamma}^{\bar{p}} S_{e}^{-1}, \quad \bar{\gamma}^{\bar{p}}\left(\bar{e}^{-1} e\right)_{\bar{p}}^{p}=S_{e}^{-1} \gamma^{p} S_{e},
$$

and

$$
\gamma^{q} L_{q}^{p}=S_{L} \gamma^{p} S_{L}^{-1}, \quad \quad \bar{\gamma}^{\bar{q}} \bar{L}_{\bar{q}}^{\bar{p}}=\left(S_{e}^{-1} S_{L} S_{e}\right)^{-1} \bar{\gamma}^{\bar{p}}\left(S_{e}^{-1} S_{L} S_{e}\right)
$$

Further, from (2.4), we get

$$
S_{L} \gamma^{(D+1)}=\operatorname{det}(L) \gamma^{(D+1)} S_{L}, \quad\left(S_{e}^{-1} S_{L} S_{e}\right) \bar{\gamma}^{(D+1)}=\operatorname{det}(\bar{L}) \bar{\gamma}^{(D+1)}\left(S_{e}^{-1} S_{L} S_{e}\right)
$$

where from (3.18),

$$
\operatorname{det}(L)=\operatorname{det}(\bar{L})^{-1}=\frac{\operatorname{det}[\mathbf{a}+\mathbf{b}(g+B)]}{\operatorname{det}[\mathbf{a}-\mathbf{b}(g-B)]},
$$

of which the value must be either +1 or -1 , since $L$ and $\bar{L}$ are local Lorentz transformations (3.19). Thus, if $\operatorname{det}(L)=+1, S_{L}$ commutes with $\gamma^{(D+1)}$. Otherwise, $\operatorname{det}(L)=-1$, they anti-commute. As we shall see in the following section 4, this gives the necessary and sufficient condition for the primed fermions to flip their chiralities under $\mathbf{O}(D, D)$ T-duality.

\section{Covariant Dirac operators}

In this section, utilizing the semi-covariant derivative in refs. [14, 18], we construct covariant Dirac operators for unprimed and primed fermions separately, and discuss the chirality change of the primed fermions under $\mathbf{O}(D, D)$ T-duality.

\subsection{Semi-covariant derivative for double-gauge symmetry: review}

By definition $[14,18]$, the semi-covariant derivative acts on a generic $\mathbf{O}(D, D)$ tensor density with weight, $\omega_{T}$, as

$$
\nabla_{C} T_{A_{1} A_{2} \cdots A_{n}}:=\partial_{C} T_{A_{1} A_{2} \cdots A_{n}}-\omega_{T} \Gamma^{B}{ }_{B C} T_{A_{1} A_{2} \cdots A_{n}}+\sum_{i=1}^{n} \Gamma_{C A_{i}}{ }^{B} T_{A_{1} \cdots A_{i-1} B A_{i+1} \cdots A_{n}},
$$

and it annihilates the pair of rank-two projections and the DFT-dilaton (and hence the NS-NS sector completely),

$$
\nabla_{A} P_{B C}=0, \quad \nabla_{A} \bar{P}_{B C}=0, \quad \nabla_{A} d:=-\frac{1}{2} e^{2 d} \nabla_{A}\left(e^{-2 d}\right)=\partial_{A} d+\frac{1}{2} \Gamma^{B}{ }_{B A}=0 .
$$

Note that the DFT-dilaton, $d$, is related to the string dilaton, $\phi$, through [11]

$$
e^{-2 d}=\sqrt{-g} e^{-2 \phi}
$$

and hence $e^{-2 d}=\sqrt{-g} e^{-2 \phi}$ is a scalar density with weight one. In fact, this is the only quantity having a nontrivial weight in this paper. 
It follows from (4.2) that, the $\mathbf{O}(D, D)$ metric is also 'flat' with respect to the semicovariant derivative,

$$
\nabla_{A} \mathcal{J}_{B C}=0
$$

which implies

$$
\Gamma_{A B C}=-\Gamma_{A C B}
$$

Further, requiring

$$
\Gamma_{A B C}+\Gamma_{B C A}+\Gamma_{C A B}=0
$$

and

$$
\mathcal{P}_{C A B}{ }^{D E F} \Gamma_{D E F}=0, \quad \overline{\mathcal{P}}_{C A B}{ }^{D E F} \Gamma_{D E F}=0,
$$

the connection is uniquely fixed to be [18]

$$
\begin{aligned}
\Gamma_{C A B}= & 2\left(P \partial_{C} P \bar{P}\right)_{[A B]}+2\left(\bar{P}_{[A}^{D} \bar{P}_{B]}^{E}-P_{[A}^{D} P_{B]}^{E}\right) \partial_{D} P_{E C} \\
& -\frac{4}{D-1}\left(\bar{P}_{C[A} \bar{P}_{B]}^{D}+P_{C[A} P_{B]}^{D}\right)\left(\partial_{D} d+\left(P \partial^{E} P \bar{P}\right)_{[E D]}\right) .
\end{aligned}
$$

In (4.7), $\mathcal{P}_{C A B}{ }^{D E F}$ and $\overline{\mathcal{P}}_{C A B}{ }^{D E F}$ are rank-six projections,

$$
\begin{aligned}
& \mathcal{P}_{C A B}{ }^{D E F}:=P_{C}{ }^{D} P_{[A}{ }^{[E} P_{B]}{ }^{F]}+\frac{2}{D-1} P_{C[A} P_{B]}{ }^{[E} P^{F] D}, \\
& \overline{\mathcal{P}}_{C A B}{ }^{D E F}:=\bar{P}_{C}{ }^{D} \bar{P}_{[A}{ }^{[E} \bar{P}_{B]}{ }^{F]}+\frac{2}{D-1} \bar{P}_{C[A} \bar{P}_{B]}{ }^{[E} \bar{P}^{F] D},
\end{aligned}
$$

that are symmetric and traceless,

$$
\begin{array}{ll}
\mathcal{P}_{C A B D E F}=\mathcal{P}_{D E F C A B}=\mathcal{P}_{C[A B] D[E F]}, & \overline{\mathcal{P}}_{C A B D E F}=\overline{\mathcal{P}}_{D E F C A B}=\overline{\mathcal{P}}_{C[A B] D[E F]}, \\
\mathcal{P}_{C A B} D E F \mathcal{P}_{D E F} G H I=\mathcal{P}_{C A B} G H I, & \overline{\mathcal{P}}_{C A B} D E F \overline{\mathcal{P}}_{D E F} G H I=\overline{\mathcal{P}}_{C A B} G H I \\
\mathcal{P}_{A B D E F}^{A}=0, \quad P^{A B} \mathcal{P}_{A B C D E F}=0, & \overline{\mathcal{P}}_{A B D E F}^{A}=0, \quad \bar{P}_{A B B} \overline{\mathcal{P}}_{A B C D E F}=0 .
\end{array}
$$

The symmetric properties, (4.5) and (4.6), enable us to replace the ordinary derivatives in the definition of the generalized Lie derivative (1.4) by our semi-covariant derivatives (4.1), i.e. $\hat{\mathcal{L}}_{X}^{\partial} \rightarrow \hat{\mathcal{L}}_{X}^{\nabla}$. The additional constraints (4.6) and (4.7) are analogue to the torsionless condition in Riemannian geometry that uniquely picks up the the Levi-Civita connection. In fact, assuming the skew-symmetric property, $\Gamma_{A B C}=-\Gamma_{A C B}$ only, the difference between $\hat{\mathcal{L}}_{X}^{\partial}$ and $\hat{\mathcal{L}}_{X}^{\nabla}$ is given by the totally anti-symmetric part of the connection,

$$
\left(\hat{\mathcal{L}}_{X}^{\nabla}-\hat{\mathcal{L}}_{X}^{\partial}\right) T_{A_{1} \cdots A_{n}}=\sum_{i=1}^{n}\left(\Gamma_{A_{i} B C}+\Gamma_{B C A_{i}}+\Gamma_{C A_{i} B}\right) X^{C} T_{A_{1} \cdots A_{i-1}}{ }^{B}{ }_{A_{i+1} \cdots A_{n}},
$$

such that this difference might be used for the definition of "torsion" [34]. However we emphasize that, the symmetric properties (4.5), (4.6) are not sufficient enough to fix the connection uniquely: the projective condition (4.7) must be also imposed.

Under the double-gauge transformations, the connection and the semi-covariant derivative transform as

$$
\begin{aligned}
\left(\delta_{X}-\hat{\mathcal{L}}_{X}\right) \Gamma_{C A B} & \equiv 2\left[(\mathcal{P}+\overline{\mathcal{P}})_{C A B}{ }^{F D E}-\delta_{C}^{F} \delta_{A}^{D} \delta_{B}^{E}\right] \partial_{F} \partial_{[D} X_{E]} \\
\left(\delta_{X}-\hat{\mathcal{L}}_{X}\right) \nabla_{C} T_{A_{1} \cdots A_{n}} & \equiv \sum_{i=1}^{n} 2(\mathcal{P}+\overline{\mathcal{P}})_{C A_{i}}{ }^{B F D E} \partial_{F} \partial_{[D} X_{E]} T_{A_{1} \cdots A_{i-1} B A_{i+1} \cdots A_{n}}
\end{aligned}
$$


Hence, they are not double-gauge covariant. We say, a tensor is double-gauge covariant if and only if its double-gauge transformation agrees with the generalized Lie derivative, i.e. ' $\delta_{X}=\hat{\mathcal{L}}_{X}$ '. Nonetheless, the characteristic feature of the semi-covariant derivative is that, combined with the projections, it can generate various fully covariant quantities, and hence the name 'semi-covariant':

$$
\begin{gathered}
P_{C}{ }^{D} \bar{P}_{A_{1}}{ }^{B_{1}} \bar{P}_{A_{2}}{ }^{B_{2}} \cdots \bar{P}_{A_{n}}{ }^{B_{n}} \nabla_{D} T_{B_{1} B_{2} \cdots B_{n}}, \\
\bar{P}_{C}{ }^{D} P_{A_{1}}{ }^{B_{1}} P_{A_{2}} B_{2} \cdots P_{A_{n}}{ }^{B_{n}} \nabla_{D} T_{B_{1} B_{2} \cdots B_{n}}, \\
P^{A B} \bar{P}_{C_{1}}{ }_{1} \bar{P}_{C_{2}}{ }^{D_{2}} \cdots \bar{P}_{C_{n}}{ }^{D_{n}} \nabla_{A} T_{B D_{1} D_{2} \cdots D_{n},}, \\
\bar{P}^{A B} P_{C_{1}}{ }^{D_{1}} P_{C_{2}} D_{2} \cdots P_{C_{n}}{ }^{D_{n}} \nabla_{A} T_{B D_{1} D_{2} \cdots D_{n},}, \\
P^{A B} \bar{P}_{C_{1}} D_{1} \bar{P}_{C_{2}} D_{2} \cdots \bar{P}_{C_{n}}{ }^{D_{n}} \nabla_{A} \nabla_{B} T_{D_{1} D_{2} \cdots D_{n}}, \\
\bar{P}^{A B} P_{C_{1}}{ }^{D_{1}} P_{C_{2}}{ }^{D_{2}} \cdots P_{C_{n}}{ }^{D_{n}} \nabla_{A} \nabla_{B} T_{D_{1} D_{2} \cdots D_{n}} .
\end{gathered}
$$

With the usual curvature,

$$
R_{C D A B}=\partial_{A} \Gamma_{B C D}-\partial_{B} \Gamma_{A C D}+\Gamma_{A C}^{E} \Gamma_{B E D}-\Gamma_{B C}{ }^{E} \Gamma_{A E D},
$$

that turns out to be double-gauge non-covariant, if we set

$$
S_{A B C D}:=\frac{1}{2}\left(R_{A B C D}+R_{C D A B}-\Gamma_{A B}^{E} \Gamma_{E C D}\right),
$$

a double-gauge covariant rank two-tensor and a double-gauge covariant scalar follow

$$
P_{I}^{A} \bar{P}_{J}^{B} S_{A B}, \quad P^{A B} S_{A B} \equiv-\bar{P}^{A B} S_{A B} .
$$

Here we put

$$
S_{A B}=S_{B A}:=S_{A C B}^{C},
$$

that turns out to be traceless,

$$
S_{A}^{A} \equiv 0 .
$$

In particular, the covariant scalar reduces to the bosonic closed string effective action upon the level matching constraint (1.1) [18],

$$
2 P^{A B} S_{A B} \equiv R_{g}+4 \square \phi-4 \partial_{\mu} \phi \partial^{\mu} \phi-\frac{1}{12} H_{\lambda \mu \nu} H^{\lambda \mu \nu} .
$$

\subsection{Unprimed Dirac operators: $\mathrm{O}(D, D)$ singlet}

For the $\mathbf{O}(D, D)$ singlet fermions, i.e.unprimed fermions, $\left(\psi_{\bar{p}}^{\alpha}, \rho^{\alpha}\right)$, we focus on the following differential operator,

$$
\mathcal{D}_{A}:=\partial_{A}+\Gamma_{A}+\Phi_{A}+\bar{\Phi}_{A}=\nabla_{A}+\Phi_{A}+\bar{\Phi}_{A}=D_{A}+\Gamma_{A},
$$

where $D_{A}$, is a local Lorenz covariant, yet double-gauge non-covariant, derivative having the connections, $\Phi_{A}$ and $\bar{\Phi}_{A}$ for $\mathbf{S O}(1, D-1)$ and $\mathbf{S O}(D-1,1)$ respectively,

$$
D_{A}:=\partial_{A}+\Phi_{A}+\bar{\Phi}_{A} .
$$


We view $\mathcal{D}_{A}$, as our 'master' unprimed, semi-covariant derivative unifying $\nabla_{A}$ and $D_{A}$. We require it to annihilate the unprimed double-vielbein and the DFT-dilaton,

$$
\begin{gathered}
\mathcal{D}_{A} V_{B p}=\partial_{A} V_{B p}+\Gamma_{A B}{ }^{C} V_{C p}+\Phi_{A p}{ }^{q} V_{B q}=0, \\
\mathcal{D}_{A} \bar{V}_{B \bar{p}}=\partial_{A} \bar{V}_{B \bar{p}}+\Gamma_{A B}{ }^{C} \bar{V}_{C \bar{p}}+\bar{\Phi}_{A \bar{p}} \bar{q} \bar{V}_{B \bar{q}}=0, \\
\mathcal{D}_{A} d=\nabla_{A} d:=-\frac{1}{2} e^{2 d} \nabla_{A}\left(e^{-2 d}\right)=\partial_{A} d+\frac{1}{2} \Gamma_{B A}^{B}=0,
\end{gathered}
$$

as well as all the constant metrics and the gamma matrices in table 2 ,

$$
\begin{aligned}
& \mathcal{D}_{A} \mathcal{J}_{B C}=0, \quad \mathcal{D}_{A} \eta_{p q}=0, \quad \mathcal{D}_{A} \bar{\eta}_{\bar{p} \bar{q}}=0, \quad \mathcal{D}_{A} C_{\alpha \beta}=0, \quad \mathcal{D}_{A} \bar{C}_{\bar{\alpha} \bar{\beta}}=0, \\
& \mathcal{D}_{A}\left(\gamma^{p}\right)^{\alpha}{ }_{\beta}=\Phi_{A}{ }^{p}{ }_{q}\left(\gamma^{q}\right)^{\alpha}{ }_{\beta}+\Phi_{A}{ }^{\alpha}{ }_{\delta}\left(\gamma^{p}\right)^{\delta}{ }_{\beta}-\left(\gamma^{p}\right)^{\alpha}{ }_{\delta} \Phi_{A}{ }_{\beta}=0, \\
& \mathcal{D}_{A}\left(\bar{\gamma}^{\bar{p}}\right)^{\bar{\alpha}}{ }_{\bar{\beta}}=\bar{\Phi}_{A} \bar{p}_{\bar{q}}\left(\bar{\gamma}^{\bar{q}}\right)^{\bar{\alpha}}{ }_{\bar{\beta}}+\bar{\Phi}_{A} \bar{\alpha}_{\bar{\delta}}\left(\bar{\gamma}^{\bar{p}}\right)^{\bar{\delta}}{ }_{\bar{\beta}}-\left(\bar{\gamma}^{\bar{p}}\right)^{\bar{\alpha}}{ }_{\bar{\delta}} \bar{\Phi}_{A} \bar{\delta}_{\bar{\beta}}=0 .
\end{aligned}
$$

It follows that

$$
\mathcal{D}_{A} P_{B C}=\nabla_{A} P_{B C}=0, \quad \mathcal{D}_{A} \bar{P}_{B C}=\nabla_{A} \bar{P}_{B C}=0,
$$

and as usual,,$^{5}$

$$
\Phi_{A p q}=-\Phi_{A q p}, \quad \bar{\Phi}_{A \bar{p} \bar{q}}=-\bar{\Phi}_{A \bar{q} \bar{p}}, \quad \Phi_{A}^{\alpha}{ }_{\beta}=\frac{1}{4} \Phi_{A p q}\left(\gamma^{p q}\right)^{\alpha}{ }_{\beta}, \quad \bar{\Phi}_{A} \bar{\alpha}_{\bar{\beta}}=\frac{1}{4} \bar{\Phi}_{A \bar{p} \bar{q}}\left(\bar{\gamma}^{\bar{p} \bar{q}}\right)^{\bar{\alpha}}{ }_{\bar{\beta}} .
$$

Specifically the spin connections are determined, from (4.22) with (4.8), by

$$
\Phi_{A p q}=V_{p}^{B} \nabla_{A} V_{B q}, \quad \bar{\Phi}_{A \bar{p} \bar{q}}=\bar{V}^{B}{ }_{\bar{p}} \nabla_{A} \bar{V}_{B \bar{q}},
$$

such that

$$
\Gamma_{A B C}=V_{B}^{p} D_{A} V_{C p}+\bar{V}_{B}^{\bar{p}} D_{A} \bar{V}_{C \bar{p}} .
$$

From the consideration of $\left[\mathcal{D}_{A}, \mathcal{D}_{B}\right] V_{C p}=0$ and $\left[\mathcal{D}_{A}, \mathcal{D}_{B}\right] \bar{V}_{C \bar{p}}=0$, we may derive the relations between the covariant scalar, $P^{A B} S_{A B}$ (4.16), and the field strengths of the local Lorentz connections,

$$
\begin{aligned}
& P^{A B} S_{A B}=F_{A B p q} V^{A p} V^{B q}-\frac{1}{2} \Gamma_{A B C} \Gamma^{A B}{ }_{D} P^{C D} \\
& \bar{P}^{A B} S_{A B}=\bar{F}_{A B \bar{p} \bar{q}} \bar{V}^{A \bar{p}} \bar{V}^{B \bar{q}}-\frac{1}{2} \Gamma_{A B C} \Gamma^{A B}{ }_{D} \bar{P}^{C D}
\end{aligned}
$$

where, in fact $P^{A B} S_{A B}=-\bar{P}^{A B} S_{A B}$ due to (4.18), and the field strengths are as usual,

$$
\begin{aligned}
& F_{A B p q}=\partial_{A} \Phi_{B p q}-\partial_{B} \Phi_{A p q}+\Phi_{A p r} \Phi_{B}^{r} q-\Phi_{B p r} \Phi_{A}^{r} q \\
& \bar{F}_{A B \bar{p} \bar{q}}=\partial_{A} \bar{\Phi}_{B \bar{p} \bar{q}}-\partial_{B} \bar{\Phi}_{A \bar{p} \bar{q}}+\bar{\Phi}_{A \bar{p} \bar{r}} \bar{\Phi}_{B}{ }_{B}^{\bar{r}} \bar{q}-\bar{\Phi}_{B \bar{p} \bar{r}} \bar{\Phi}_{A}{ }^{\bar{r}} \bar{q}
\end{aligned}
$$

\footnotetext{
${ }^{5}$ Here, for simplicity, we omit the possibility of adding a central term to the spin connections, i.e.

$$
\Phi_{A}^{\alpha}{ }_{\beta}=\frac{1}{4} \Phi_{A p q}\left(\gamma^{p q}\right)^{\alpha}{ }_{\beta}+c \times \delta_{\beta}^{\alpha} .
$$
}


Though $\Phi_{A p q}$ and $\bar{\Phi}_{A \bar{p} \bar{q}}$ are not double-gauge covariant from (4.12), ${ }^{6}$

$$
\begin{aligned}
& \left(\delta_{X}-\hat{\mathcal{L}}_{X}\right) \Phi_{A p q} \equiv 2 \mathcal{P}_{A B C}{ }^{D E F} \partial_{D} \partial_{[E} X_{F]} V^{B}{ }_{p} V^{C}{ }_{q}, \\
& \left(\delta_{X}-\hat{\mathcal{L}}_{X}\right) \bar{\Phi}_{A \bar{p} \bar{q}} \equiv 2 \overline{\mathcal{P}}_{A B C}{ }^{D E F} \partial_{D} \partial_{[E} X_{F]} \bar{V}^{B}{ }_{\bar{p}} \bar{V}_{\bar{q}}^{C},
\end{aligned}
$$

with (4.9), the followings are so, i.e. ' $\delta_{X} \equiv \hat{\mathcal{L}}_{X}$ ',

$$
\bar{P}_{A}^{B} \Phi_{B p q}, \quad P_{A}^{B} \bar{\Phi}_{B \bar{p} \bar{q}}, \quad \Phi_{A[p q} V_{r]}^{A}, \quad \bar{\Phi}_{A[\bar{p} \bar{q}} \bar{V}_{\bar{r}]}^{A}, \quad \Phi_{A p q} V^{A p}, \quad \bar{\Phi}_{A \bar{p} \bar{q}} \bar{V}^{A \bar{p}} .
$$

This generalizes our earlier results in [18] where only the first two in (4.30) were identified.

After all, the fully covariant unprimed Dirac operators, with respect to all the symmetries in table 1 , are as follows

$$
\gamma^{A} \mathcal{D}_{A} \rho, \quad \gamma^{A} \mathcal{D}_{A} \psi_{\bar{p}}, \quad \bar{V}_{\bar{p}}^{A} \mathcal{D}_{A} \rho, \quad \bar{V}^{A \bar{p}} \mathcal{D}_{A} \psi_{\bar{p}}=\mathcal{D}_{A} \psi^{A}
$$

Here we set for simplicity,

$$
\psi_{A}:=\bar{V}_{A}^{\bar{p}} \psi_{\bar{p}}, \quad \gamma^{A}:=V_{p}^{A} \gamma^{p},
$$

such that

$$
\bar{V}_{\bar{p}}^{A} \psi_{A}=\psi_{\bar{p}}, \quad\left\{\gamma^{A}, \gamma^{B}\right\}=2 P^{A B}
$$

Writing explicitly,

$$
\begin{aligned}
\mathcal{D}_{A} \rho & =D_{A} \rho=\left(\partial_{A}+\frac{1}{4} \Phi_{A p q} \gamma^{p q}\right) \rho \\
\mathcal{D}_{A} \psi_{\bar{p}} & =D_{A} \psi_{\bar{p}}=\left(\partial_{A}+\frac{1}{4} \Phi_{A p q} \gamma^{p q}\right) \psi_{\bar{p}}+\bar{\Phi}_{A \bar{p}}{ }^{\bar{q}} \psi_{\bar{q}}, \\
\mathcal{D}_{A} \psi_{B} & =\left(\partial_{A}+\frac{1}{4} \Phi_{A p q} \gamma^{p q}\right) \psi_{B}+\Gamma_{A B}{ }^{C} \psi_{C} .
\end{aligned}
$$

As all the associated fields in (4.31) are unprimed (unprimed double-vielbein and unprimed fermions), all the unprimed Dirac operators are $\mathbf{O}(D, D)$ singlets.

\subsection{Primed Dirac operators}

In this subsection, we construct the fully covariant, 'primed' Dirac operators for the $\mathbf{O}(D, D)$ non-singlet fermions, i.e. the primed fermions, $\left(\psi_{\bar{p}}^{\prime}, \rho^{\alpha}\right)$. As the analysis is parallel to the previous subsection on the unprimed fermions, we skip the details and present only the main results.

The primed master semi-covariant derivative is

$$
\mathcal{D}_{A}^{\prime}:=\partial_{A}+\Gamma_{A}+\Phi_{A}^{\prime}+\bar{\Phi}_{A}^{\prime}=\nabla_{A}+\Phi_{A}^{\prime}+\bar{\Phi}_{A}^{\prime}=D_{A}^{\prime}+\Gamma_{A},
$$

where

$$
D_{A}^{\prime}:=\partial_{A}+\Phi_{A}^{\prime}+\bar{\Phi}_{A}^{\prime},
$$

\footnotetext{
${ }^{6}$ For double-gauge covariant yet $\mathbf{O}(D, D)$ non-covariant connections for the local Lorentz symmetries, see our earlier work [18]. In this paper, instead we focus on the double-gauge semi-covariant and $\mathbf{O}(D, D)$ covariant connections for the local Lorentz symmetries.
} 
and

$$
\begin{aligned}
& \Phi_{A p q}^{\prime}=-\bar{V}_{B p}^{\prime} \nabla_{A} \bar{V}^{\prime B}{ }_{q}, \quad \bar{\Phi}_{A}^{\prime}{ }_{A \bar{p} \bar{q}}=-V_{B \bar{p}}^{\prime} \nabla_{A} V^{\prime B}{ }_{\bar{q}}, \\
& \Gamma_{A B C}=-V^{\prime}{ }_{B} \bar{p} D_{A}^{\prime} V^{\prime}{ }_{C \bar{p}}-\bar{V}_{B}^{\prime}{ }_{B}^{p} D_{A}^{\prime} \bar{V}^{\prime}{ }_{C p} .
\end{aligned}
$$

It satisfies

$$
\begin{aligned}
\mathcal{D}_{A}^{\prime} V_{B \bar{p}}^{\prime} & =0, \quad \mathcal{D}_{A}^{\prime} \bar{V}_{B p}^{\prime}=0, \quad \mathcal{D}_{A}^{\prime} d=\nabla_{A} d=\partial_{A} d+\frac{1}{2} \Gamma_{B A}^{B}=0, \\
\mathcal{D}_{A}^{\prime} P_{B C}=\nabla_{A} P_{B C} & =0, \quad \mathcal{D}_{A}^{\prime} \bar{P}_{B C}=\nabla_{A} \bar{P}_{B C}=0,
\end{aligned}
$$

and like (4.23),

$$
\begin{aligned}
& \mathcal{D}_{A}^{\prime} \mathcal{J}_{B C}=0, \quad \mathcal{D}_{A}^{\prime} \eta_{p q}=0, \quad \mathcal{D}_{A}^{\prime} \bar{\eta}_{\bar{p} \bar{q}}=0 \\
& \mathcal{D}_{A}^{\prime} C_{\alpha \beta}=0, \quad \mathcal{D}_{A}^{\prime} \bar{C}_{\bar{\alpha} \bar{\beta}}=0, \quad \mathcal{D}_{A}^{\prime}\left(\gamma^{p}\right)^{\alpha}{ }_{\beta}=0, \quad \mathcal{D}_{A}^{\prime}\left(\bar{\gamma}^{\bar{p}}\right)^{\bar{\alpha}}{ }_{\bar{\beta}}=0 .
\end{aligned}
$$

Further, in analogy to (4.28), we have

$$
\begin{aligned}
& P^{A B} S_{A B}=-\bar{F}_{A B \bar{p} \bar{q}} V^{\prime A \bar{p}} V^{\prime B \bar{q}}-\frac{1}{2} \Gamma_{A B C} \Gamma^{A B}{ }_{D} P^{C D}, \\
& \bar{P}^{A B} S_{A B}=-F^{\prime}{ }_{A B p q} \bar{V}^{\prime A p} \bar{V}^{\prime B q}-\frac{1}{2} \Gamma_{A B C} \Gamma^{A B}{ }_{D} \bar{P}^{C D}
\end{aligned}
$$

where the primed field strengths are, in an identical fashion to (4.29),

$$
F_{A B}^{\prime}=\partial_{A} \Phi_{B}^{\prime}-\partial_{B} \Phi_{A}^{\prime}+\left[\Phi_{A}^{\prime}, \Phi_{B}^{\prime}\right], \quad \bar{F}_{A B}^{\prime}=\partial_{A} \bar{\Phi}_{B}^{\prime}-\partial_{B} \bar{\Phi}_{A}^{\prime}+\left[\bar{\Phi}_{A}^{\prime}, \bar{\Phi}_{B}{ }_{B}\right]
$$

Finally, the fully covariant primed Dirac operators are

$$
\gamma^{\prime A} \mathcal{D}_{A}^{\prime} \rho^{\prime}, \quad \quad \gamma^{\prime A} \mathcal{D}_{A}^{\prime} \psi_{\bar{p}}^{\prime}, \quad V^{\prime A}{ }_{\bar{p}} \mathcal{D}_{A}^{\prime} \rho^{\prime}, \quad V^{\prime A \bar{p}} \mathcal{D}_{A}^{\prime} \psi^{\prime} \bar{p}_{\bar{p}}=\mathcal{D}_{A}^{\prime} \psi^{\prime A}
$$

Here we set for simplicity,

$$
\psi_{A}^{\prime}:=V_{A}^{\prime}{ }^{\bar{p}} \psi_{\bar{p}}^{\prime}, \quad \gamma^{\prime A}:=\bar{V}^{\prime A}{ }_{p} \gamma^{p},
$$

such that

$$
V^{\prime A}{ }_{\bar{p}} \psi_{A}^{\prime}=-\psi_{\bar{p}}^{\prime}, \quad\left\{\gamma^{\prime A}, \gamma^{\prime B}\right\}=-2 \bar{P}^{A B} .
$$

Writing explicitly we have

$$
\begin{aligned}
\mathcal{D}_{A}^{\prime} \rho^{\prime} & =D_{A}^{\prime} \rho^{\prime}=\left(\partial_{A}+\frac{1}{4} \Phi_{A p q}^{\prime} \gamma^{p q}\right) \rho^{\prime}, \\
\mathcal{D}_{A}^{\prime} \psi_{\bar{p}}^{\prime} & =D_{A}^{\prime} \psi_{\bar{p}}^{\prime}=\left(\partial_{A}+\frac{1}{4} \Phi_{A p q}^{\prime} \gamma^{p q}\right) \psi_{\bar{p}}^{\prime}+\bar{\Phi}^{\prime}{ }_{A \bar{p}}{ }^{\bar{q}} \psi_{\bar{q}}^{\prime}, \\
\mathcal{D}_{A}^{\prime} \psi_{B}^{\prime} & =\left(\partial_{A}+\frac{1}{4} \Phi^{\prime}{ }_{A p q} \gamma^{p q}\right) \psi_{B}^{\prime}+\Gamma_{A B}{ }^{C} \psi_{C}^{\prime} .
\end{aligned}
$$




\subsection{Chirality change under $\mathrm{O}(D, D)$ T-duality}

In order to discuss the $\mathbf{O}(D, D)$ transformations of the primed fermions, we first recall the $\mathbf{O}(D, D)$ transformation rule of the primed double-vielbein (3.21),

$$
V_{A \bar{p}}^{\prime} \quad \longrightarrow \quad M_{A}^{B} \bar{L}_{\bar{p}}^{\bar{q}} V_{B \bar{q}}^{\prime}, \quad \bar{V}_{A p}^{\prime} \longrightarrow M_{A}^{B} L_{p}^{q} \bar{V}_{B q}^{\prime}
$$

where, from (3.14) and (3.18), the $\mathbf{O}(D, D)$ group element and the associated local Lorentz transformations are given by

$$
M_{A}{ }^{B}=\left(\begin{array}{cc}
\mathbf{a}^{\mu}{ }_{\nu} & \mathbf{b}^{\mu \sigma} \\
\mathbf{c}_{\rho \nu} & \mathbf{d}_{\rho}{ }^{\sigma}
\end{array}\right), \quad \begin{aligned}
& L=e^{-1}\left[\mathbf{a}^{t}+(g-B) \mathbf{b}^{t}\right]\left[\mathbf{a}^{t}-(g+B) \mathbf{b}^{t}\right]^{-1} e, \\
& \bar{L}=\left(\bar{e}^{-1} e\right) L^{-1}\left(e^{-1} \bar{e}\right) .
\end{aligned}
$$

We also recall the covariance of the gamma matrices (3.23),

$$
\gamma^{q} S_{L} L_{q}^{p}=S_{L} \gamma^{p}
$$

It is then clear that, with the full covariance of the primed Dirac operators (4.42), the primed fermions transform under $\mathbf{O}(D, D)$ T-duality as follows,

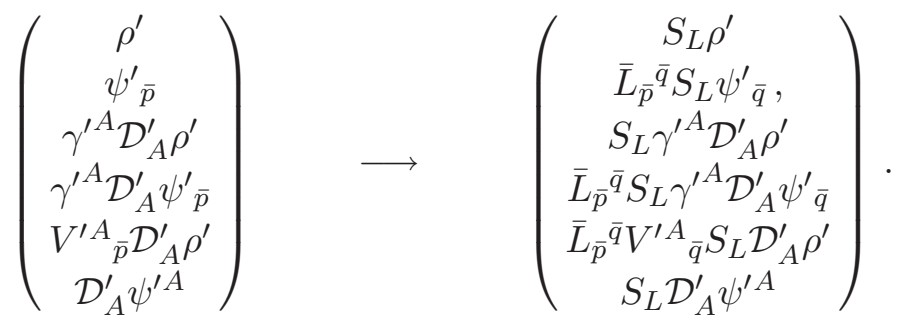

Thus, from (3.24) and (3.25),

$$
\gamma^{(D+1)} S_{L}=\operatorname{det}(L) S_{L} \gamma^{(D+1)}, \quad \operatorname{det}(L)=\frac{\operatorname{det}[\mathbf{a}+\mathbf{b}(g+B)]}{\operatorname{det}[\mathbf{a}-\mathbf{b}(g-B)]}= \pm 1
$$

when $\operatorname{det}(L)=-1$, the primed fermions flip their chiralities. Otherwise not.

For example, on a flat background $(g=\eta, B=0)$, we may set both $\mathbf{a}$ and $\mathbf{b} g$ to be diagonal with the eigenvalues, zero or one only, in an exclusive manner such that $\mathbf{a}+\mathbf{b} g=1$. This choice corresponds to the usual discrete T-duality along toroidal directions. In this case, we get $\operatorname{det}(L)=(-1)^{\sharp a}$ where $\sharp_{\mathrm{a}}$ counts the number of zero eigenvalues in the matrix, a, and hence the number of toroidal directions on which T-duality is performed. Thus, our formula is consistent with the well-known knowledge that performing odd number of T-duality on flat backgrounds exchanges type IIA and IIB superstrings.

\subsection{Reduction to $D$ dimension}

Upon the level matching constraint (1.1), with the explicit forms of the doublevielbeins (3.6), (3.10), the covariant DFT Dirac operators (4.31), (4.42) reduce to more 
familiar $D$-dimensional expressions within the Riemannian setup,

$$
\begin{aligned}
\sqrt{2} \gamma^{A} \mathcal{D}_{A} \rho & \equiv \gamma^{m}\left(\partial_{m} \rho+\frac{1}{4} \omega_{m n p} \gamma^{n p} \rho+\frac{1}{24} H_{m n p} \gamma^{n p} \rho-\partial_{m} \phi \rho\right) \\
\sqrt{2} \gamma^{A} \mathcal{D}_{A} \psi_{\bar{p}} & \equiv \gamma^{m}\left(\partial_{m} \psi_{\bar{p}}+\frac{1}{4} \omega_{m n p} \gamma^{n p} \psi_{\bar{p}}+\bar{\omega}_{m \bar{p} \bar{q}} \psi^{\bar{q}}+\frac{1}{24} H_{m n p} \gamma^{n p} \psi_{\bar{p}}+\frac{1}{2} H_{m \bar{p} \bar{q}} \psi^{\bar{q}}-\partial_{m} \phi \psi_{\bar{p}}\right) \\
\sqrt{2} \bar{V}^{A}{ }_{\bar{p}} \mathcal{D}_{A} \rho & \equiv \partial_{\bar{p}} \rho+\frac{1}{4} \omega_{\bar{p} q r} \gamma^{q r} \rho+\frac{1}{8} H_{\bar{p} q r} \gamma^{q r} \rho \\
\sqrt{2} \mathcal{D}_{A} \psi^{A} & \equiv \partial^{\bar{p}} \psi_{\bar{p}}+\frac{1}{4} \omega_{\bar{p} q r} \gamma^{q r} \psi^{\bar{p}}+\bar{\omega}^{\bar{p}} \overline{\bar{p}} \psi^{\bar{q}}+\frac{1}{8} H_{\bar{p} q r} \gamma^{q r} \psi^{\bar{p}}-2 \partial_{\bar{p}} \phi \psi^{\bar{p}} \\
\sqrt{2} \gamma^{\prime A} \mathcal{D}_{A}^{\prime} \rho^{\prime} & \equiv \gamma^{m}\left(\partial_{m} \rho^{\prime}+\frac{1}{4} \omega_{m n p} \gamma^{n p} \rho^{\prime}-\frac{1}{24} H_{m n p} \gamma^{n p} \rho^{\prime}-\partial_{m} \phi \rho^{\prime}\right) \\
\sqrt{2} \gamma^{\prime A} \mathcal{D}_{A}^{\prime} \psi_{\bar{p}}^{\prime} & \equiv \gamma^{m}\left(\partial_{m} \psi_{\bar{p}}^{\prime}+\frac{1}{4} \omega_{m n p} \gamma^{n p} \psi_{\bar{p}}^{\prime}+\bar{\omega}_{m \bar{p}} \bar{q} \psi_{\bar{q}}^{\prime}-\frac{1}{24} H_{m n p} \gamma^{n p} \psi_{\bar{p}}^{\prime}-\frac{1}{2} H_{m \bar{p} \bar{q}} \psi^{\prime \bar{q}}-\partial_{m} \phi \psi_{\bar{p}}^{\prime}\right) \\
\sqrt{2} V^{\prime A}{ }_{\bar{p}} \mathcal{D}_{A}^{\prime} \rho^{\prime} & \equiv \partial_{\bar{p}} \rho^{\prime}+\frac{1}{4} \omega_{\bar{p} q r} \gamma^{q r} \rho^{\prime}-\frac{1}{8} H_{\bar{p} q r} \gamma^{q r} \rho^{\prime}, \\
\sqrt{2} \mathcal{D}_{A}^{\prime} \psi^{\prime A} & \equiv \partial^{\bar{p}} \psi_{\bar{p}}^{\prime}+\frac{1}{4} \omega^{\bar{p}}{ }_{q r} \gamma^{q r} \psi_{\bar{p}}^{\prime}+\bar{\omega}^{\bar{p}} \overline{\bar{p} \bar{q}} \psi^{\prime \bar{q}}-\frac{1}{8} H_{\bar{p} q r} \gamma^{q r} \psi^{\prime \bar{p}}-2 \partial_{\bar{p}} \phi \psi^{\prime \bar{p}}
\end{aligned}
$$

where, with the $D$-dimensional standard diffeomorphism covariant derivative, $\nabla_{\mu}$, we set $\partial_{p}=\left(e^{-1}\right)_{p}{ }^{\mu} \partial_{\mu}, \partial_{\bar{p}}=\left(\bar{e}^{-1}\right)_{\bar{p}}{ }^{\mu} \partial_{\mu}, \omega_{\mu p q}=\left(e^{-1}\right)_{p}{ }^{\nu} \nabla_{\mu} e_{\nu q}, \bar{\omega}_{\mu \bar{p} \bar{q}}=\left(\bar{e}^{-1}\right)_{\bar{p}}{ }^{\nu} \nabla_{\mu} \bar{e}_{\nu \bar{q}}$, etc.

In fact, the above expressions are precisely what appear in type IIA and IIB supergravities [34], where $\psi_{\bar{p}}$ and $\psi^{\prime} \bar{p}$ are gravitinos in string frame, while $\rho$ and $\rho^{\prime}$ are 'DFT-dilatinos' corresponding to the superpartner of the DFT-dilaton, $d=\phi-\frac{1}{2} \ln \sqrt{-g}$.

\section{Summary and comments}

In summary, based on the stringy differential geometry that is characterized by the semicovariant derivative [18], we have incorporated fermions, like gravitino and dilatino, into double field theory in a manifestly covariant manner with regard to all the symmetries in table 1, i.e. $\mathbf{O}(D, D)$ T-duality, double-gauge symmetry and a pair of local Lorentz symmetries. We have shown that in general there are two types of fermions in double field theory: $\mathbf{O}(D, D)$ singlet and non-singlet (unprimed and primed). For each type, we have constructed relevant covariant Dirac operators, (4.31) and (4.42). Especially, we have derived a necessary and sufficient condition for the primed fermions to flip their chiralities under $\mathbf{O}(D, D)$ T-duality (4.50), that depends on both the $\mathbf{O}(D, D)$ group element and the background fields.

In this paper, we have chosen the primed fermions, $\left(\rho^{\prime \alpha}, \psi^{\prime} \overline{\bar{p}}\right)$, to carry the same local Lorentz indices as the unprimed fermions, $\left(\rho^{\alpha}, \psi_{\bar{p}}^{\alpha}\right)$. The alternative choice is also possible: If we let the primed fermions have the opposite local Lorentz structure like $\left(\rho^{\prime \bar{\alpha}}, \psi^{\prime} \bar{\alpha}\right)$, their 
fully covariant Dirac operators are, with $\bar{\gamma}^{A}:=V^{\prime}{ }_{\bar{p}} \bar{\gamma}^{\bar{p}}$,

$$
\begin{aligned}
\sqrt{2} \bar{\gamma}^{\prime A} \mathcal{D}_{A}^{\prime} \rho^{\prime} & \equiv \bar{\gamma}^{\bar{m}}\left(\partial_{\bar{m}} \rho^{\prime}+\frac{1}{4} \bar{\omega}_{\bar{m} \bar{p} \bar{q}} \bar{\gamma} \bar{p}^{\bar{p}} \rho^{\prime}-\frac{1}{24} H_{\bar{m} \bar{p} \bar{q}} \bar{\gamma}^{\bar{p} \bar{q}} \rho^{\prime}-\partial_{\bar{m}} \phi \rho^{\prime}\right) \\
\sqrt{2} \bar{\gamma}^{\prime A} \mathcal{D}_{A}^{\prime} \psi_{p}^{\prime} & \equiv \bar{\gamma}^{\bar{m}}\left(\partial_{\bar{m}} \psi_{p}^{\prime}+\frac{1}{4} \bar{\omega}_{\bar{m} \bar{p} \bar{q}} \bar{\gamma}^{\bar{p} \bar{q}} \psi_{p}^{\prime}+\omega_{\bar{m} p}{ }^{q} \psi^{\prime}{ }_{q}-\frac{1}{24} H_{\bar{m} \bar{p} \bar{q}} \bar{\gamma}^{\bar{p} \bar{q}} \psi_{p}^{\prime}-\frac{1}{2} H_{\bar{m} p q} \psi^{\prime q}-\partial_{\bar{m}} \phi \psi_{p}^{\prime}\right), \\
\sqrt{2} \bar{V}^{\prime A}{ }_{p} \mathcal{D}_{A}^{\prime} \rho^{\prime} & \equiv \partial_{p} \rho^{\prime}+\frac{1}{4} \bar{\omega}_{p \bar{p} \bar{q}} \bar{\gamma}^{\bar{p} \bar{q}} \rho^{\prime}-\frac{1}{8} H_{p \bar{p} \bar{q}} \bar{\gamma}^{\bar{p} \bar{q}} \rho^{\prime}, \\
\sqrt{2} \bar{V}^{\prime A p} D_{A}^{\prime} \psi_{p}^{\prime} & \equiv \partial^{p} \psi_{p}^{\prime}+\frac{1}{4} \bar{\omega}^{p}{ }_{\bar{p} \bar{q} \bar{\gamma}}^{\bar{p} \bar{q}} \psi_{p}^{\prime}+\omega^{p}{ }_{p q} \psi^{\prime q}-\frac{1}{8} H_{p \bar{p} \bar{q}} \bar{\gamma}^{\bar{p} \bar{q}} \psi^{\prime p}-2 \partial_{p} \phi \psi^{\prime p}
\end{aligned}
$$

However, these seem irrelevant to type IIA and IIB supergravity (e.g. see eq. (2.8) in [34]).

It is worth while to note that, the distinction between the primed and unprimed doublevielbein is arbitrary: if we set one to be $\mathbf{O}(D, D)$ vector, like (3.13), then the other is not a vector anymore, like (3.21). Thus, $\mathbf{O}(D, D)$ may act on the unprimed fermions nontrivially while leaving the primed fermions singlet. This seems to imply the doubling of $\mathbf{O}(D, D)$ structures. Further investigation is required.

So far, we have focused on the gravitational interpretation of the unprimed and primed fermions. However, we may also regard $\rho$ or $\rho^{\prime}$ as gaugino and couple them to the YangMills double field theory [16].

Up to the RR sector (for related works see e.g. [22, 34, 38]), the unifying supersymmetric double field theory reformulation of type IIA and IIB supergravities will, when constructed [39], contain the following leading order terms (see also [28]),

$e^{-2 d}\left(P^{A B} S_{A B}+\bar{\rho} \gamma^{A} \mathcal{D}_{A} \rho+2 \bar{\psi}^{A} \mathcal{D}_{A} \rho+\bar{\psi}^{A} \gamma^{B} \mathcal{D}_{B} \psi_{A}+\bar{\rho}^{\prime} \gamma^{\prime A} \mathcal{D}_{A}^{\prime} \rho^{\prime}+2 \bar{\psi}^{\prime A} \mathcal{D}_{A}^{\prime} \rho^{\prime}+\bar{\psi}^{A} \gamma^{\prime B} \mathcal{D}_{B}^{\prime} \psi^{\prime}{ }_{A}\right)$.

In particular, the complete supersymmetric double field theory will manifest not only $\mathbf{O}(D, D)$ T-duality and double-gauge symmetry, but also a pair of local Lorentz symmetries. It will be of interest to identify the pair of local Lorentz symmetries directly from the string worldsheet or $\mathcal{M}$-theory points of view [19-21, 40-47].

\section{Acknowledgments}

We wish to thank David Berman and Daniel Waldram for discussion. The work was supported by the National Research Foundation of Korea (NRF) grants funded by the Korea government (MEST) with the Grant No. 2005-0049409 (CQUeST) and No. 20100002980. The work by IJ is partially supported by NRF though the Korea-CERN theory collaboration.

Open Access. This article is distributed under the terms of the Creative Commons Attribution Noncommercial License which permits any noncommercial use, distribution, and reproduction in any medium, provided the original author(s) and source are credited.

\section{References}

[1] T. Buscher, Quantum corrections and extended supersymmetry in new $\sigma$-models, Phys. Lett. B 159 (1985) 127 [INSPIRE]. 
[2] T. Buscher, A Symmetry of the String Background Field Equations, Phys. Lett. B 194 (1987) 59 [inSPIRE].

[3] T. Buscher, Path Integral Derivation of Quantum Duality in Nonlinear $\sigma$-models, Phys. Lett. B 201 (1988) 466 [inSPIRE].

[4] A. Giveon, E. Rabinovici and G. Veneziano, Duality in String Background Space, Nucl. Phys. B 322 (1989) 167 [inSPIRE].

[5] A.A. Tseytlin, Duality symmetric formulation of string world sheet dynamics, Phys. Lett. B 242 (1990) 163 [INSPIRE].

[6] A.A. Tseytlin, Duality symmetric closed string theory and interacting chiral scalars, Nucl. Phys. B 350 (1991) 395 [inSPIRE].

[7] W. Siegel, Two vierbein formalism for string inspired axionic gravity, Phys. Rev. D 47 (1993) 5453 [hep-th/9302036] [INSPIRE].

[8] W. Siegel, Superspace duality in low-energy superstrings, Phys. Rev. D 48 (1993) 2826 [hep-th/9305073] [INSPIRE].

[9] C. Hull and B. Zwiebach, Double Field Theory, JHEP 09 (2009) 099 [arXiv:0904.4664] [INSPIRE].

[10] C. Hull and B. Zwiebach, The Gauge algebra of double field theory and Courant brackets, JHEP 09 (2009) 090 [arXiv:0908.1792] [INSPIRE].

[11] O. Hohm, C. Hull and B. Zwiebach, Background independent action for double field theory, JHEP 07 (2010) 016 [arXiv: 1003.5027] [INSPIRE].

[12] O. Hohm, C. Hull and B. Zwiebach, Generalized metric formulation of double field theory, JHEP 08 (2010) 008 [arXiv: 1006.4823] [INSPIRE].

[13] S.K. Kwak, Invariances and Equations of Motion in Double Field Theory, JHEP 10 (2010) 047 [arXiv: 1008.2746] [INSPIRE].

[14] I. Jeon, K. Lee and J.-H. Park, Differential geometry with a projection: Application to double field theory, JHEP 04 (2011) 014 [arXiv: 1011.1324] [INSPIRE].

[15] O. Hohm and S.K. Kwak, Frame-like Geometry of Double Field Theory, J. Phys. A A 44 (2011) 085404 [arXiv:1011.4101] [InSPIRE].

[16] I. Jeon, K. Lee and J.-H. Park, Double field formulation of Yang-Mills theory, Phys. Lett. B 701 (2011) 260 [arXiv:1102.0419] [InSPIRE].

[17] O. Hohm and S.K. Kwak, Double Field Theory Formulation of Heterotic Strings, JHEP 06 (2011) 096 [arXiv:1103.2136] [INSPIRE].

[18] I. Jeon, K. Lee and J.-H. Park, Stringy differential geometry, beyond Riemann, Phys. Rev. D 84 (2011) 044022 [arXiv: 1105.6294] [INSPIRE].

[19] N.B. Copland, Connecting T-duality invariant theories, Nucl. Phys. B 854 (2012) 575 [arXiv:1106.1888] [INSPIRE].

[20] D.C. Thompson, Duality Invariance: From M-theory to Double Field Theory, JHEP 08 (2011) 125 [arXiv: 1106.4036] [INSPIRE].

[21] C. Albertsson, S.-H. Dai, P.-W. Kao and F.-L. Lin, Double Field Theory for Double D-branes, JHEP 09 (2011) 025 [arXiv:1107.0876] [INSPIRE]. 
[22] O. Hohm, S.K. Kwak and B. Zwiebach, Double Field Theory of Type II Strings, JHEP 09 (2011) 013 [arXiv: 1107.0008] [INSPIRE].

[23] O. Hohm and S.K. Kwak, Massive Type II in Double Field Theory, arXiv:1108.4937 [INSPIRE].

[24] B. Zwiebach, Double Field Theory, T-duality and Courant Brackets, arXiv:1109.1782 [INSPIRE].

[25] E. Cremmer, B. Julia and J. Scherk, Supergravity Theory in Eleven-Dimensions, Phys. Lett. B 76 (1978) 409 [INSPIRE].

[26] E. Cremmer and B. Julia, The $N=8$ Supergravity Theory. 1. The Lagrangian, Phys. Lett. B 80 (1978) 48 [inSPIRE].

[27] J. Maharana and J.H. Schwarz, Noncompact symmetries in string theory, Nucl. Phys. B 390 (1993) 3 [hep-th/9207016] [InSPIRE].

[28] A. Kleinschmidt and H. Nicolai, $E_{10}$ and $S O(9,9)$ invariant supergravity, JHEP 07 (2004) 041 [hep-th/0407101] [INSPIRE].

[29] T. Courant, Dirac Manifolds, Trans. Am. Math. Soc. 319 (1990) 631.

[30] N. Hitchin, Generalized Calabi-Yau manifolds, Quart. J. Math. Oxford Ser. 54 (2003) 281 [math/0209099] [INSPIRE].

[31] N. Hitchin, Lectures on generalized geometry, arXiv:1008.0973 [INSPIRE].

[32] M. Gualtieri, Generalized complex geometry, math/0401221 [INSPIRE].

[33] M. Graña, R. Minasian, M. Petrini and D. Waldram, T-duality, Generalized Geometry and Non-Geometric Backgrounds, JHEP 04 (2009) 075 [arXiv:0807.4527] [InSPIRE].

[34] A. Coimbra, C. Strickland-Constable and D. Waldram, Supergravity as Generalised Geometry I: Type II Theories, arXiv:1107.1733 [INSPIRE].

[35] S. Hassan, $O(d, d: R)$ deformations of complex structures and extended world sheet supersymmetry, Nucl. Phys. B 454 (1995) 86 [hep-th/9408060] [INSPIRE].

[36] S. Hassan, T duality, space-time spinors and RR fields in curved backgrounds, Nucl. Phys. B 568 (2000) 145 [hep-th/9907152] [INSPIRE].

[37] S. Hassan, $S O(d, d)$ transformations of Ramond-Ramond fields and space-time spinors, Nucl. Phys. B 583 (2000) 431 [hep-th/9912236] [INSPIRE].

[38] M. Fukuma, T. Oota and H. Tanaka, Comments on T dualities of Ramond-Ramond potentials on tori, Prog. Theor. Phys. 103 (2000) 425 [hep-th/9907132] [INSPIRE].

[39] I. Jeon, K. Lee and J.-H. Park, Supersymmetric Double Field Theory: Stringy Reformulation of Supergravity, in progress.

[40] C. Hull, A Geometry for non-geometric string backgrounds, JHEP 10 (2005) 065 [hep-th/0406102] [INSPIRE].

[41] C.M. Hull, Doubled Geometry and T-Folds, JHEP 07 (2007) 080 [hep-th/0605149] [INSPIRE].

[42] D.S. Berman, N.B. Copland and D.C. Thompson, Background Field Equations for the Duality Symmetric String, Nucl. Phys. B 791 (2008) 175 [arXiv: 0708. 2267] [InSPIRE]. 
[43] D.S. Berman and D.C. Thompson, Duality Symmetric Strings, Dilatons and O(d,d) Effective Actions, Phys. Lett. B 662 (2008) 279 [arXiv:0712.1121] [INSPIRE].

[44] D.S. Berman and M.J. Perry, Generalized Geometry and M-theory, JHEP 06 (2011) 074 [arXiv: 1008.1763] [INSPIRE].

[45] D.S. Berman, H. Godazgar and M.J. Perry, SO(5,5) duality in M-theory and generalized geometry, Phys. Lett. B 700 (2011) 65 [arXiv:1103.5733] [INSPIRE].

[46] N. Kan, K. Kobayashi and K. Shiraishi, Equations of Motion in Double Field Theory: From particles to scale factors, arXiv:1108.5795 [INSPIRE].

[47] G. Aldazabal, W. Baron, D. Marques and C. Núñez, The effective action of Double Field Theory, arXiv: 1109.0290 [INSPIRE]. 